This item was submitted to Loughborough's Research Repository by the author.

Items in Figshare are protected by copyright, with all rights reserved, unless otherwise indicated.

\title{
Abnormal events detection based on RP and inception network using distributed optical fiber perimeter system
}

PLEASE CITE THE PUBLISHED VERSION

https://doi.org/10.1016/j.optlaseng.2020.106377

PUBLISHER

Elsevier

VERSION

AM (Accepted Manuscript)

\section{PUBLISHER STATEMENT}

This paper was accepted for publication in the journal Optics and Lasers in Engineering and the definitive published version is available at https://doi.org/10.1016/j.optlaseng.2020.106377

LICENCE

CC BY-NC-ND 4.0

\section{REPOSITORY RECORD}

Lyu, Chengang, Jianying Jiang, Baihua Li, Ziqiang Huo, and Jiachen Yang. 2020. "Abnormal Events Detection Based on RP and Inception Network Using Distributed Optical Fiber Perimeter System”. Loughborough University. https://hdl.handle.net/2134/12964901.v1. 


\title{
Abnormal events detection based on RP and inception network using distributed optical fiber perimeter system
}

\author{
Chengang Lyu, ${ }^{1,}{ }^{*}$ Jianying JIANg, ${ }^{1}$ Baihua LI, ${ }^{2}$ ZiQIANg Huo, ${ }^{1}$ AND \\ JIACHEN YANG ${ }^{1}$ \\ ${ }^{1}$ Electrical and Information Engineering, Tianjin University, Tianjin, 300072, China \\ ${ }^{2}$ Department of Computer Science, Loughborough University, Loughborough LE11 3TU, U.K. \\ *lvchengang@tju.edu.cn
}

\begin{abstract}
For establishing an accurate and reliable distributed optical fiber perimeter security system, this paper proposes a novel abnormity detection solution to security using Recurrent Plot (RP) and deep learning technology. Take advantage of the temporal correlation of intrusion signals, we encode the sensing signals into two-dimensional images through the RP algorithm. The RP algorithm can extract the motion characteristics of the signal from the complex time series, and it is robust to instrument noise. These encoded image signatures can reveal the deeper temporal correlation of the intrusion signals' motion. After that, Inception network can adaptively extract the features of these images to complete the accurate identification of a series of noisy intrusion signals. We conducted experiments on three most frequent natural events and three representative man-made intrusion events, including heavy rain, light rain, wind blowing, treading, slapping, and impacting. The results show that the detection accuracy has reached $99.7 \%$. This method can achieve $0.35 \mathrm{~s}$ real-time detection in the online detection of abnormal events while ensuring accuracy, providing a new intrusion pattern identification idea for perimeter security.
\end{abstract}

Keywords: Distributed optical fiber sensing, events detection, inception network, Recurrent Plot.

\section{Introduction}

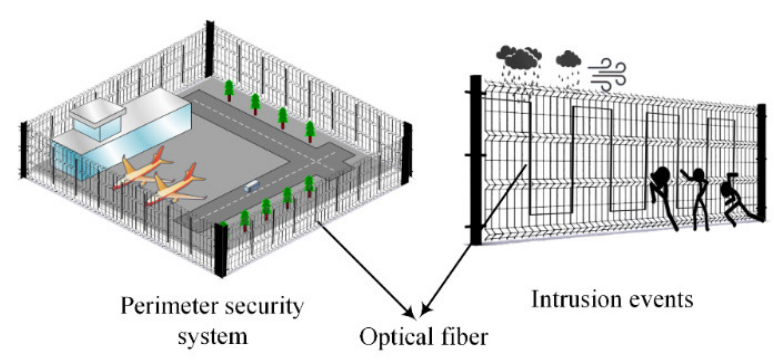

Fig. 1. Distributed optical fiber perimeter security system and intrusion events.

Distributed optical fiber perimeter security system is a safety monitoring system constructed by optical fiber technology at the border of the security zone. It is widely used in the security of community, industry, military and other areas[1-5]. The system has strong environmental adaptability and high sensitivity, which can realize long-distance and wide-range monitoring [6]. As shown in Fig. 1, with distributed optical fiber perimeter security system, different natural or man-made intrusion event signals are collected at any time. And these events should be identified and responded accurately and quickly. At present, the hardware system of optical fiber sensor for intrusion events monitoring has been becoming matured [7]. However, the identification method of intrusion event pattern is still an open problem to be explored.

Time-frequency based analysis method is commonly used for intrusion signal pattern 
identification. Wu et al. [8] used wavelet decomposition method combined with probabilistic neural network to analyze non-stationary signals and extract local features of the signals. However, it requires to select the appropriate Basic Wavelet and set feasible decomposition layers, which is not easily adaptive to different intrusion events. Jiang et al. [9] proposed a neural network identification algorithm based on Hilbert Huang transform (HHT). The HHT has the advantage of wavelet decomposition and overcomes the difficulty of choosing Basic Wavelet, whereas it is easy to cause mode mixing and edge effect, and the calculation cost is high. Liang et al. [10] used the short time maximum frequency as the feature to draw macroenvelopes in order to classify the signal according to the similarity of the envelope. It simplifies the identification process and speeds up the calculation. Unfortunately, when the difference between signal envelope is not obvious, the identification is easy to be confused. Liu et al. [11] combined the autoregressive modeling coefficient (AR) and used the zero-crossing rate (ZCR) to construct a concise eigenvector. They adopted the AdaBoost technique to synthesize multiple SVM classifier results. However, due to the performance of SVM and also the accuracy of hand-crafted features, the accuracy of identification is low.

In recent years, Deep Learning (DL) has achieved tremendous results in the field of pattern identification. Especially the Convolutional Neural Network (CNN), which shows a huge advantage in image pattern feature extraction. So, the method of transforming a onedimensional (1D) signal into a two-dimensional (2D) image and then taking advantage of CNN network has become a new idea for pattern identification of intrusion signals [12]. For example, Wang et al. [13] used the short-time Fourier transform to construct a spatial time-frequency spectrum image, and used dual path network (DPN) to automatically learn features for intrusion identification. Recently, we have studied intrusion identification method based on Gramian Angular Field (GAF) image coding and CNN [14]. These methods verify the effectiveness of the combination of signal-encoded image and CNN.

In actual application, in addition to always existed various environmental noise and instrument noise, there are also many occasional intrusion events. These occasional intrusion events are the focus of our research, including natural intrusions and man-made intrusions. These intrusion patterns produce nonlinear signals with similar frequency information. Traditional time-frequency methods are not suitable for the analysis of these signals. In the experiment, we found that the motion of the 1D signal can reflect the characteristics of the intrusion pattern. And the motion of the signal can be seen as its amplitude fluctuating up and down with time. Different pattern has different motion characteristics which can be the basis of intrusion pattern identification.

According to the above, this paper proposes an intrusion events identification method based on Recurrent Plot (RP) and inception network. This method uses RP algorithm to encode the intrusion signal into image while extracting the unique motion characteristics of each intrusion pattern. Then combined with DL inception-v3 network to achieve accurate identification of the intrusion signal in an end-to-end manner. RP algorithm is robust to noise and it can efficiently encode images. These encoded image signatures can reveal the deeper temporal correlation of the intrusion signals' motion. Combined with the advantages of deep learning, this paper can provide an accurate and fast intrusion pattern identification method, and provide a new idea for perimeter security intrusion pattern identification.

\section{Principles}

Overall structure of this paper is shown in Fig. 2. Step A: collecting intrusion signals by the distributed optical fiber dual Mach-Zehnder perimeter security system. Step B: encoding the 1D intrusion signals into 2D images by RP algorithm. Step C: using inception network to identify intrusion events. 

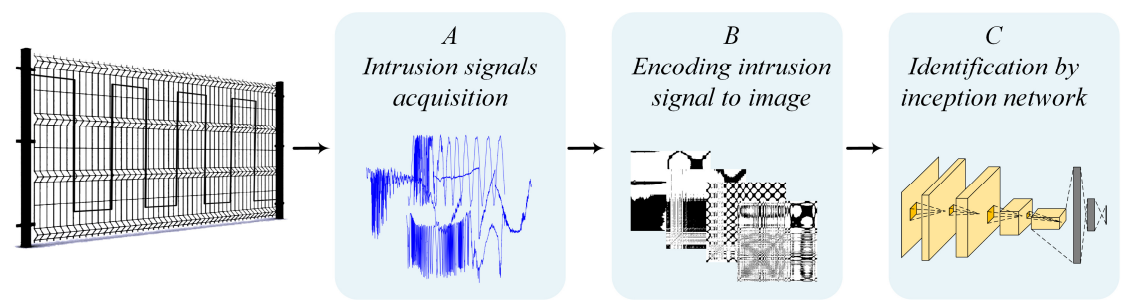

Fig. 2. Overview of overall framework.

\subsection{Intrusion signals acquisition}

Among all kinds of distributed optical fiber sensing technologies, the dual Mach-Zehnder interferometer (DMZI) is widely used in the perimeter security field. It has the advantages of high sensitivity, wide monitoring range, and location capability [6, 15-17]. In this experiment, we setup a DMZI system to collect the intrusion signals, and its structure is shown in Fig. 3.

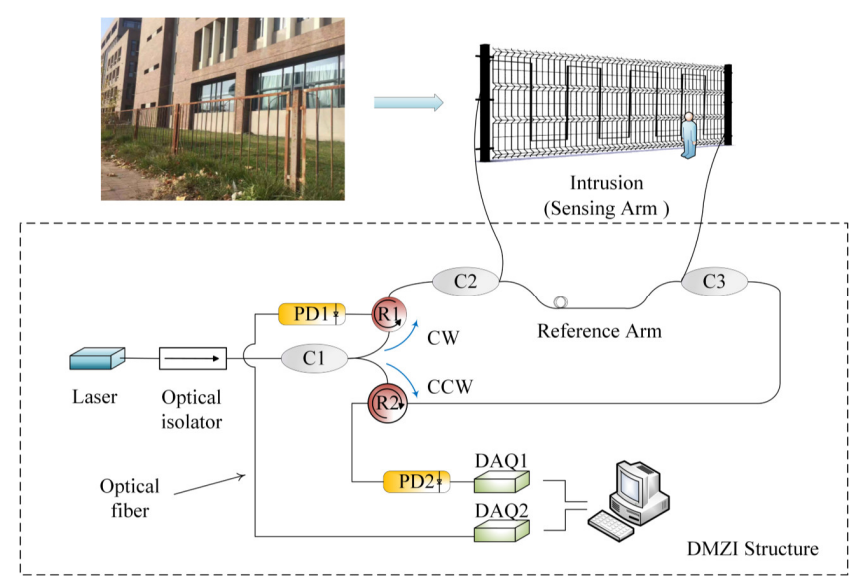

Fig. 3. DMZI structure. C1, C2, C3: fiber coupler; R1, R2: optical circulator. PD1, PD2: Photodetector. DAQ1, DAQ2: data acquisition card. CW: R1-C2-C3-R2. CCW: R2-C3-C2-R1.

The continuous narrow-band laser beam with wavelength of $1550 \mathrm{~nm}$ passes through an optical isolator, which prevents unwanted feedback into the laser source. Then through the coupler $\mathrm{C}$, the light is equally split into two paths: clockwise $(\mathrm{CW})$ and counter-clockwise $(\mathrm{CCW})$, which simultaneously propagate in both the sensing arm and the reference arm. The lights interfere at the coupler (C2 or $\mathrm{C} 3$ ) respectively. Once intrusion event occurs on the sensing arm, the transmitted light in the sensing fiber will produce a phase change, resulting in a corresponding change in the output intensity of the PD. The two intrusion signals detected by PD1 and PD2 have the same pattern, but there is phase difference due to different optical path. Therefore, event location can be done by calculating optical path difference.

Using sensing optical fiber can quickly collect massive data, providing a reliable source of data for the establishment of intrusion event datasets.

\subsection{Encoding intrusion signal to image}

Experimental research shows that intrusion signals are often non-stationary and non-linear, and they have similar frequency information, which make the identification of the intrusion signal more difficult. However, the motion of the signal can reflect the characteristics of the intrusion pattern. These characteristics can be the basis for identification. The change of the signal with 
time will not rise or fall without limit. It will always rise and fall repeatedly to show a periodic or irregular cycle state. The RP can extract this motion characteristic from the trends and laws of the signal over time [18]. Moreover, this trend of motion is not easy to be covered by noise, making the algorithm more robust to noise.

$\mathrm{RP}$ is a visualization algorithm to analyze the chaos and non-stationarity of the time series [19-22].The first step of the RP is to reconstruct the phase space. Each phase point in the traditional time domain space can only represent the current state. However, in the phase space, the phase point can not only indicate the current state of the signal, but also the future motion trend of the signal [23]. Through phase space reconstruction, the motion of the signal with time is described as the trajectory of the phase point. Then, according to the distance between the phase points, we can judge the similarity between the motion states represented by the phase points. The motion characteristics of signal are extracted according to the motion state similarity. The overall process is shown in the Fig. 4.

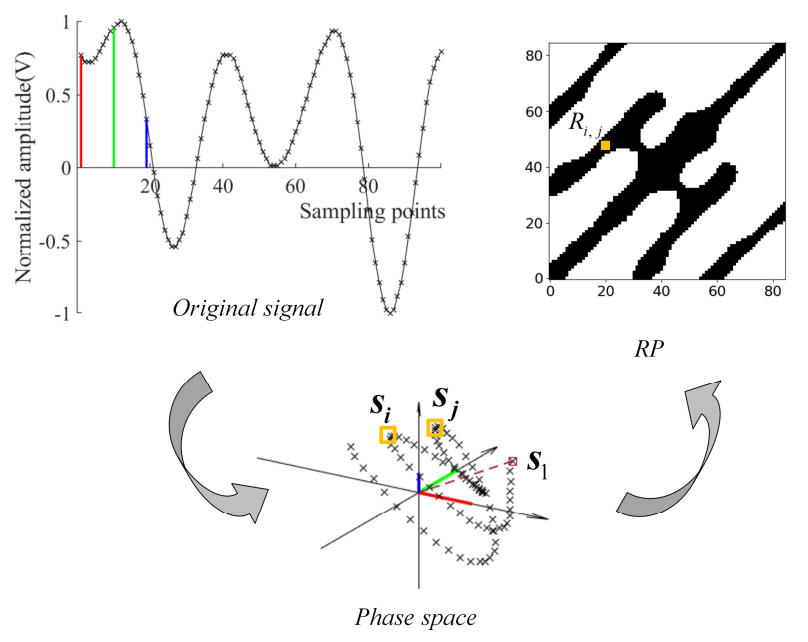

Fig. 4. From time series signal to recurrence plot $(m=3, \tau=9, n=100)$.

Select the dimension $m$ and time delay $\tau$ to perform phase space reconstruction on the 1D time series $X=\left\{x_{1}, x_{2}, \ldots, x_{i}, \ldots, x_{n}\right\}$, where $n$ is the sampling point number of the signal. We can obtain the reconstructed vector set $\{\boldsymbol{S}\}$ :

$\boldsymbol{s}_{i}=\left(x_{i}, x_{i}+\tau, \ldots, x_{i}+(m-1) \tau\right), i=1,2, \ldots, N$

where $N=n-(m-1) \tau$. The distance between two points in the phase space is:

$D_{i, j}=\left\|\boldsymbol{s}_{i}-\boldsymbol{s}_{j}\right\|, i, j=1,2, \ldots, N$

where $\|\cdot\|$ is the Euclidean norm. Then choose the appropriate threshold $\varepsilon$ to obtain the Rmatrix:

$R_{i, j}=\theta\left(\varepsilon-D_{i, j}\right)$

where $\theta(\cdot)$ is the Heaviside function and its expression is: 
$\theta(t)= \begin{cases}1 & t \geq 0 \\ 0 & t<0\end{cases}$

The R-matrix calculated according to Eq. (3) generates a 2D image signatures including motion features of the signal. R-matrix is a matrix containing only two values of 0 and 1 , which generates a binary texture image. Because of $R_{i, j}=R_{j, i}$, the generated image is symmetric about the diagonal. The RP can visually display the motion state of the intrusion signal. If the distance between the two phase points $\boldsymbol{s}_{\boldsymbol{i}}$ and $\boldsymbol{s}_{\boldsymbol{j}}$ is less than the threshold value $\varepsilon$, it is proved that the motion states of the two points are similar. The black point represents this phenomenon, otherwise it is a white point, as shown in Fig. 4.

The threshold $\varepsilon$ is used to measure the similarity of motion states. If the selected $\varepsilon$ is too small, there may be almost no black points in R-matrix, and we cannot know any information about the intrusion signal. On the other hand, if the selected $\varepsilon$ is too large, almost every point is close to other points, which results in a large number of false motion state similarities. Therefore, we must find a compromise $\varepsilon$ value. The choice of threshold $\varepsilon$ usually depends on some "rules of thumb", such as the density of recurrence points and the ratio of the maximum distance [24]. Considering that noise may distort any existing structure in RPs, this paper chooses $30 \%$ recurrence point density as the threshold.

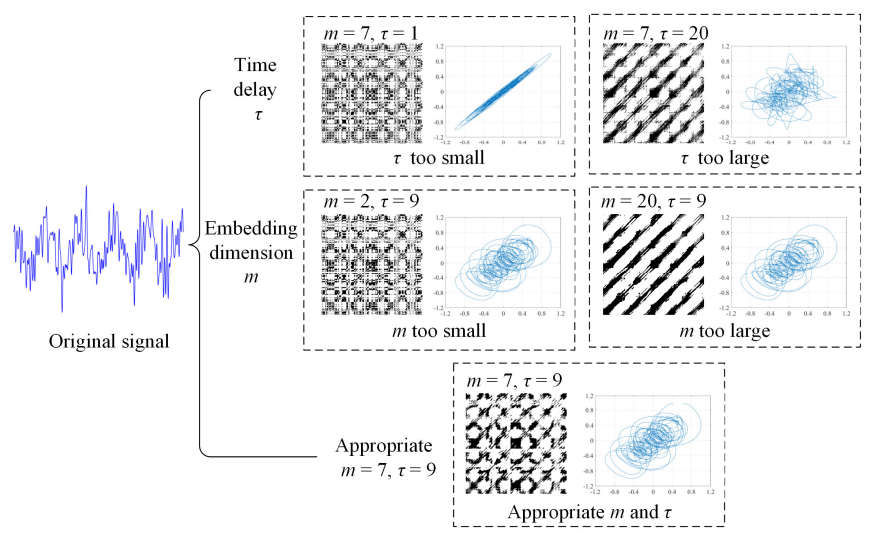

Fig. 5. Results of different parameters.

The keys to generate RPs are the choice of dimension $m$ and time delay $\tau$. As shown in Fig. 5, when $\tau$ is too small, the reconstructed phase space does not expand, but when $\tau$ is too large, the correlation between spatial points can be affected. If the $m$ selection is too small, false nearest neighbors (FNN) will be generated. That is, two points that are not adjacent in the high-dimensional space become adjacent points when they are projected into the lowdimensional space. When the selected embedding dimension $m$ is too high, the original random sequence generates some line segments with periodic regularity. So, the line segment generated due to the high embedding dimension may cover the original real feature line segment, resulting in erroneous conclusions. Moreover, too high embedding dimension will increase the amount of calculation, resulting in slower running speed. Hence, it is necessary to select the appropriate time delay and embedding dimension to reconstruct the phase space, as shown in Fig. 5. In experiment of this paper, we choose embedding dimension $m=4$, time delay $\tau=5$, and sampling points number of the signal $n=2000$ in order to reflect the motion 
characteristics of intrusion signals and ensure the computational efficiency.

$\mathrm{RP}$ is used to describe the signal phase space trajectory, that is, the motion characteristics of the signal. Its typical pattern is related to the specific motion behavior of the signal, showing large-scale global and small-scale local features [25].

The global structure of RP can be classified in four types: drift, homogeneous, periodic, and disrupted, as shown in Fig. 6. Drift structure indicates that the motion state changes slowly and vibration trajectory of the time series does not return to its original position over time. Homogeneous structure indicates that the sequence is relatively stable and random. Periodic structure indicates the cyclicities in the process, in which the distance between diagonal segments parallel to the main diagonal line $\left(R_{i, i}=1\right)$ corresponds to the period, and the node corresponds to peak or trough. Disrupted structure indicates abrupt motion changes in the time series.
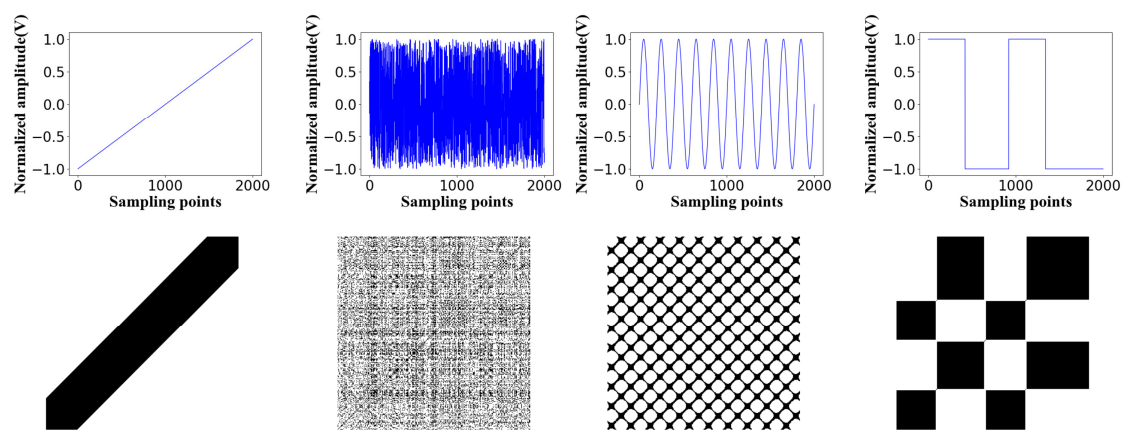

Fig. 6. RP of typical sequences ( $m=4, \tau=5, n=2000)$. Top row: time series. Bottom row: correspond RPs.

The local features of RP can be classified in three types: isolated single point, diagonal oblique lines, vertical and horizontal lines. Isolated single point indicates that the motion state lasts for a short time and mutates in the next second. Diagonal oblique line illustrates the similarity and periodicity between motion states. Vertical and horizontal line indicates that the motion state has hardly changed during the period.

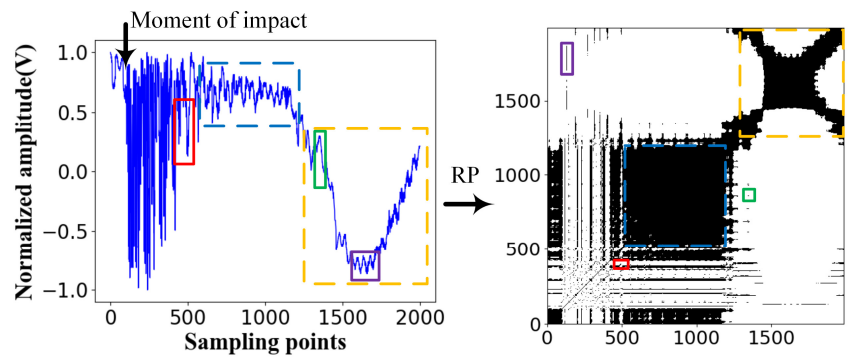

Fig. 7. Large-scale global features (dotted part) and small-scale local features (solid part) of intrusion signal's RP $(m=4, \tau=5, n=2000)$. The same color represents the corresponding part before and after RP conversion.

Take the experiment signal (Impacting) as an example, shown in Fig. 7. The dotted and solid part are the large-scale global and small-scale local feature of the intrusion events, respectively.

RPs contain a wealth of information, whose different overall global structures and detailed local features can qualitatively describe complex time series motion states. Intrusion signals 
have different motion characteristics, and their RPs have unique features, thus enabling the identification of intrusion signals.

\subsection{Identification by inception network}

Due to the development of computer technology and the advent of the era of big data, deep learning has been greatly developed. It has been successfully applied to real-time speech translation, natural language processing (NLP), object recognition in computer vision, synthetic painting, driverless vehicle and autopilot, medical applications etc. [26-29]. CNN applied to images can learn low-level simple features such as color and edges, as well as extract high-level complex texture and semantic features to form complete discriminative feature vectors. Using CNN to identify RPs, we can not only grasp the difference between intrusion signals from the whole, but also capture the features with signal details.

The neural network is enlightened by the central nervous system of the human brain, and the development of machine vision is often inspired by human visual processing. Human vision usually observes images from different scales to obtain multi-scale features. Therefore, if the neural network can observe images from different scales, it can obtain more abundant feature information. As shown in Fig. 7, the RP of intrusion signal has large-scale global and smallscale local features, using multi-scale method can get better event features and improve the identification accuracy.

This paper chooses the inception-v3 network for identification of intrusion signals, and its structure is shown in Fig. 8.

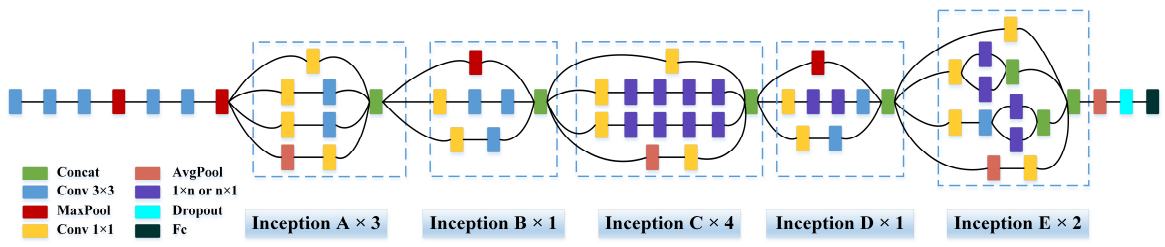

Fig. 8. Inception-v3 structure.

An important parameter influencing feature extraction in CNN is the size of the receptive field. As shown in Fig. 9, Inception modular structure contains parallel different sizes convolution kernels to provide receptive fields of different scales [30]. It does not need to manually consider the size of the convolution kernel, and can observe and mix the features of the intrusion events on multiple spatial scale.

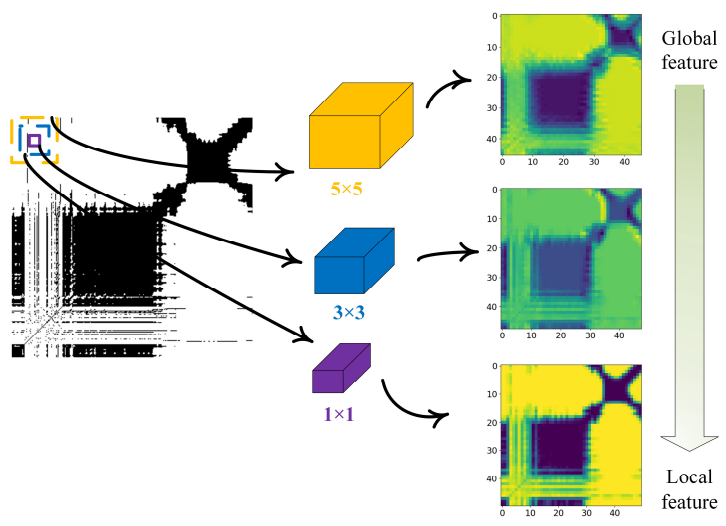

Fig. 9. Inception modular structure. 


\section{Experiment results and discussion}

The overall framework of the experiment is shown in Fig. 10. The intrusion signals were collected by the sensing optical fiber fixed on the fence. Using the RP algorithm, the acquired common intrusion signals were encoded into $2 \mathrm{D}$ images and input into the inception-v 3 for training and test.

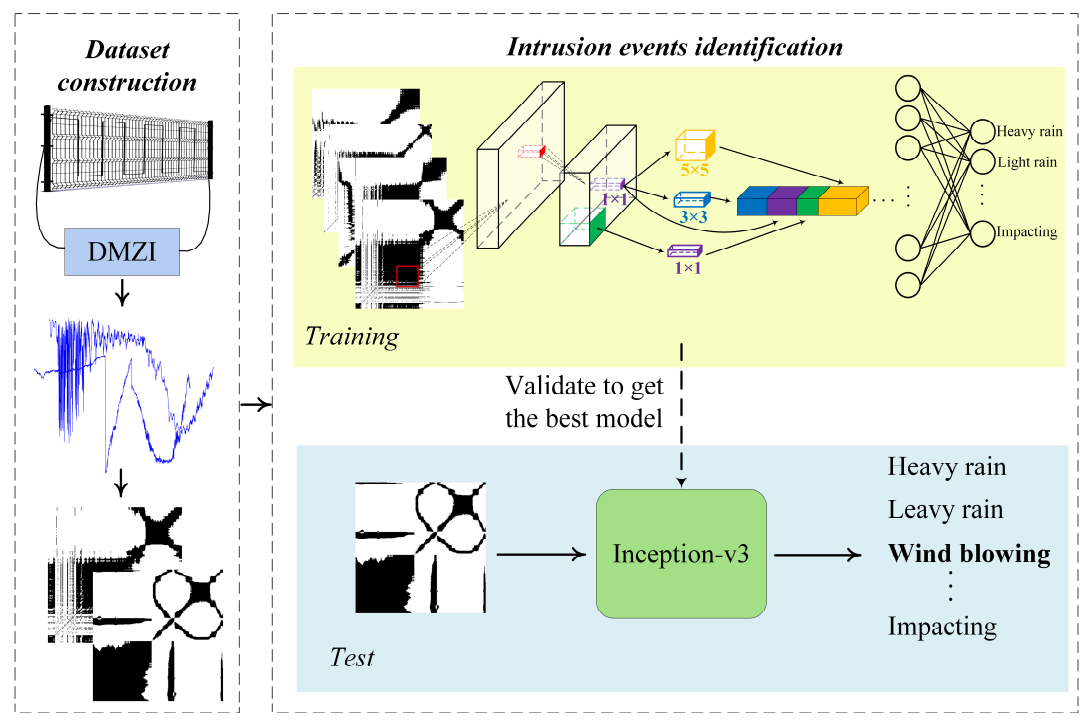

Fig. 10. Overall experimental framework.

\subsection{Dataset construction}

In the experiment, the sensing optical fiber were fixed on the fence for intrusion signal acquisition. Acquisition system is shown in Fig. 3. The laser uses Denselight Semiconductor DL-BF11-CLS. It is a single-frequency laser, and the continuous output power is $3-20 \mathrm{~mW}$. The sensing optical cable is G.652 single-mode 1-core armored-cable-based fiber which is widely used in the field of interferometric optical fiber sensing. The length of the monitored fence is about $100 \mathrm{~m}$, and the length of sensor is about $2 \mathrm{~km}$. The PD uses Thorlabs DET01CFC InGaAs photo-detector. The wavelength range is 800 to $1700 \mathrm{~nm}$ and peak response is $0.95 \mathrm{~A} / \mathrm{W}$. The DAQ is NI PCI6010 which has 16 analog inputs at up to $200 \mathrm{kS} / \mathrm{s}$.

This paper collected six typical intrusion signals: heavy rain, light rain, wind blowing, treading, slapping, and impacting. According to the intrusion events characteristics, the data sampling rate was set to be $2 \mathrm{kHz}$ so the sampling time of a frame signal was set to be $1 \mathrm{~s}$.

Selected 2000 normalized sampling points as one intrusion data, chosen embedding dimension $m=4$, time delay $\tau=5$, performed the RP conversion. A total of 7200 images of size $1985 \times 1985$ were generated as datasets, parts of intrusion signals and conversion results are demonstrated in Fig. 11. 


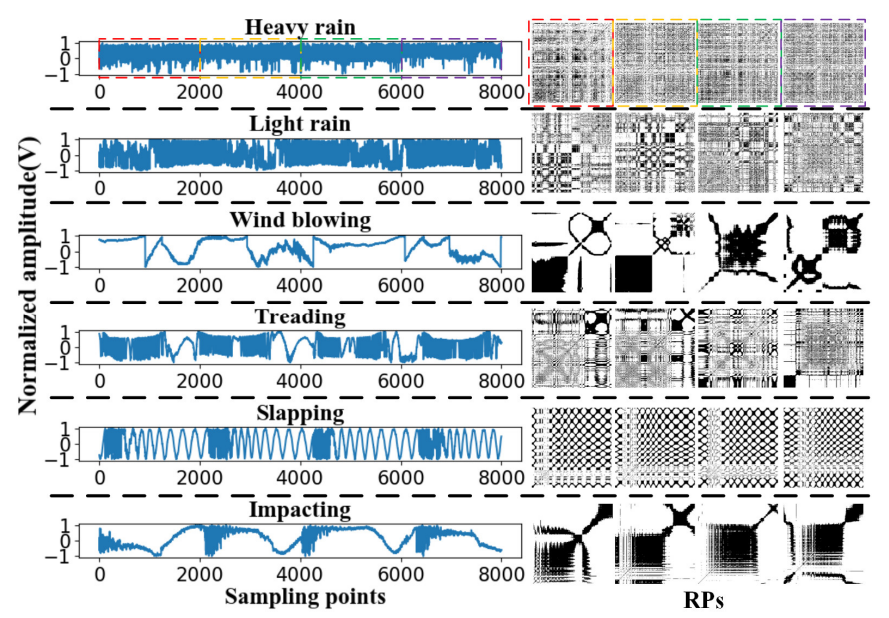

Fig. 11. Intrusion signals and converted images.

From the global features, heavy rain, light rain, and treading signals can be divided into homogeneous structures. Treading signal also contains partially drift structure. Wind blowing and impacting signals appear as disrupted structures. Slapping signal appears as periodic structure. From the local features, heavy rain signal is dense, long-lasting and non-intermittent. Light rain signal will cause black and white clumps due to the sudden decrease of amplitude during the intermittent phase. Treading signal contains more oblique and vertical segments. Wind blowing signal changes suddenly and irregularly, but impacting signal changes dramatically only at the moment of impact. So, the black and white blocks formed by the impacting signal are rougher.

It should be noted that the RP has no special requirements on the length and stability of the signals, and is insensitive to white noise. In the process of building datasets, the RPs generated before and after denoising have little difference, as shown in Fig. 12. Whether the RPs are denoised or not has little effect on the subsequent identification. Hence, it is not necessary to denoise the collected intrusion signals, which makes the identification simpler and faster.

(a)

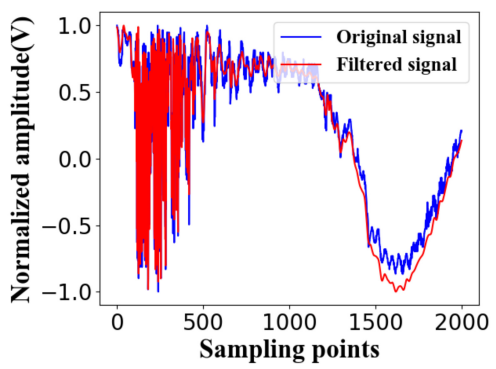

(b)

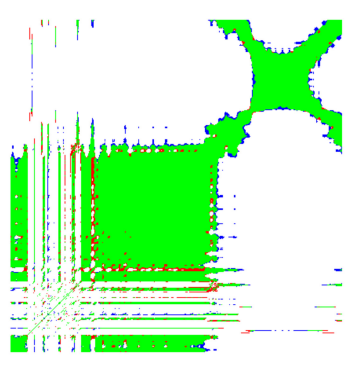

Fig. 12. Comparison before and after denoising. (a) Shows the time series waveform of the collected impacting signal, wherein the blue is before denoising and red is after wavelet denoising. (b) Comparison of RPs before and after denoising, green is the common part, blue is only the part before denoising, and red is only the part after denoising.

\subsection{Intrusion events identification}

We assume that the probability of all intrusion events occurring is the same. In the monitoring stage, we pay the same attention to all intrusion events. Hence, in order to better analyze the 
data, identify the intrusion events, this paper selected balanced data for training. According to the ratio of 3:1:1, randomly selected 4320 images from the generated RPs for training, 1440 images for validation, and 1440 images for test.

This paper chosen the inception-v3 network. In order to prevent overfitting and speed up the training, we used the method of transfer learning to train the neural network [31]. As shown in Fig. 13, we loaded the weight parameters of the pre-trained inception-v3 network, adjusted the original 1000 class classification task to 6 classes for retraining. Using transfer learning, the knowledge of the images learned by the original network in ImageNet 1000 is applied to the identification of intrusion images.

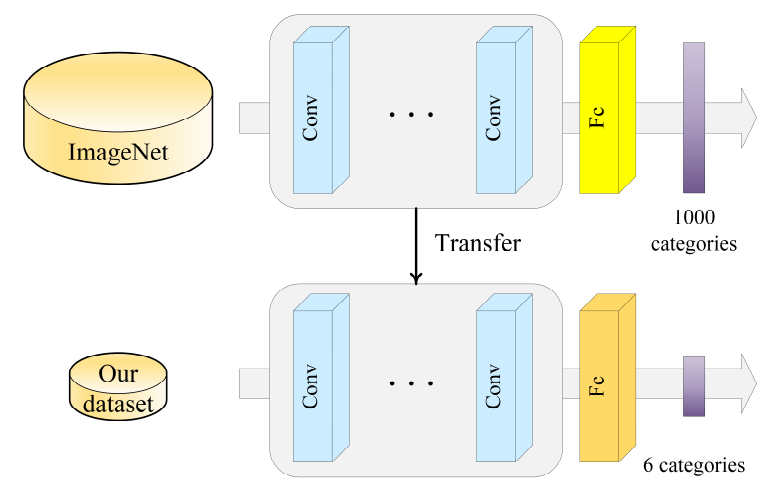

Fig. 13. Transfer learning.

Resized images to $299 \times 299$ using bilinear interpolation to reduce the input parameters of the network. The bilinear interpolation uses four points in the original image to calculate one point in the new image. Linear interpolation is performed in both the $\mathrm{x}$ direction and the $\mathrm{y}$ direction, the image quality after scaling is high. Images were input into the fine-tuned network for training. In this experiment, stochastic gradient descent (SGD) was used for parameter update. The initial value of learning rate was fixed at $1 \times 10^{-4}$, and the learning rate decay was set to fall to $80 \%$ of the original learning rate at 5th, 10th, 20th, 40th, 80th, 160th iterations. The batch size, weight decay, and number of iterations were set to $16,10^{-4}$, and 200 , respectively. Selected the cross-entropy loss function and the parameters were optimized by Adam algorithm $(\beta 1=0.9, \beta 2=0.99)$. RP conversion was implemented at Intel(R) Xeon(R) CPU@ 2.30GHz, Tesla P100. The training was implemented on a server with Intel Xeon E52620 v4 CPU @ 2.10 GHz, 128GB RAM, GeForce GTX TITAN Xp and CentOS Linux 7.6 with deep learning framework PyTorch.

\section{Results and discussion}

The results of using transfer learning and direct training are shown in Fig. 14. Fine-tuning the pre-trained network makes the training time of the target network shorter, the convergence speed faster and the weight parameters more accurate. 

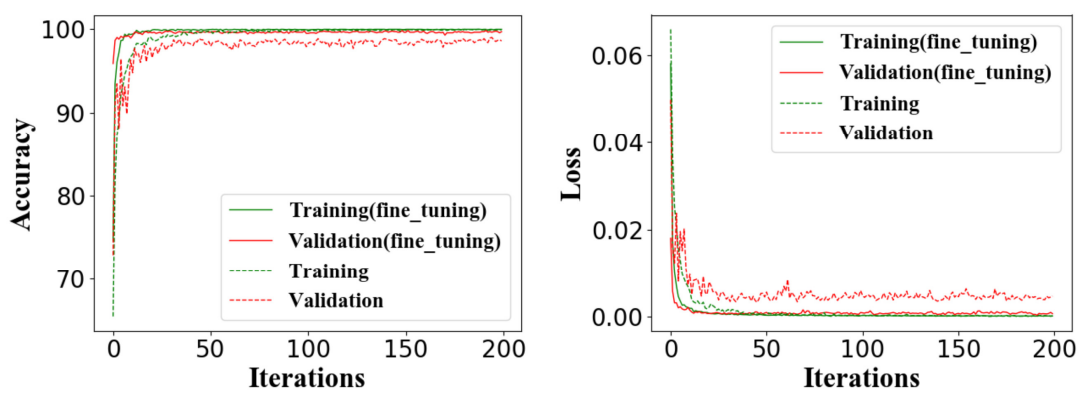

Fig. 14. Training and validation results.

In this experiment, precision and recall are used for model performance analysis. Precision is the percentage of positive sample that is currently classified correctly. Recall is the proportion of real positive samples in all positive samples that are currently assigned to the positive sample category. F1-score $=2 \times$ precision $/($ precision + recall $)$ is the comprehensive evaluation index of precision and recall.

Table 1 shows the identification results of the six intrusion events, with an average accuracy rate of $99.7 \%$, and the F1-scores are all superior to 0.99 . The confusion matrix demonstrates the same results as we analyzed above. The heavy rain and light rain signals are similar. Therefore, the densest light rain signal can lead to misjudgment.

Table 1. Identification results.

\begin{tabular}{|c|c|c|c|c|c|c|c|c|c|c|}
\hline \multirow{2}{*}{$\begin{array}{l}\text { identified } \\
\text { classes }\end{array}$} & \multicolumn{6}{|c|}{ Real classes } & \multirow{2}{*}{ Recall } & \multirow{2}{*}{ Precision } & \multirow{2}{*}{$\begin{array}{l}\text { F1- } \\
\text { Score }\end{array}$} & \multirow{2}{*}{$\begin{array}{c}\text { Accuracy } \\
(\%)\end{array}$} \\
\hline & 0 & 1 & 2 & 3 & 4 & 5 & & & & \\
\hline 0 Heavy rain & 240 & 4 & 0 & 0 & 0 & 0 & 1.000 & 0.984 & 0.992 & 100 \\
\hline 1 Light rain & 0 & 236 & 0 & 0 & 0 & 0 & 0.983 & 1.000 & 0.991 & 98.3 \\
\hline 2 Wind blowing & 0 & 0 & 240 & 0 & 0 & 0 & 1.000 & 1.000 & 1.000 & 100 \\
\hline 3 Treading & 0 & 0 & 0 & 240 & 0 & 0 & 1.000 & 1.000 & 1.000 & 100 \\
\hline 4 Slapping & 0 & 0 & 0 & 0 & 240 & 0 & 1.000 & 1.000 & 1.000 & 100 \\
\hline 5 Impacting & 0 & 0 & 0 & 0 & 0 & 240 & 1.000 & 1.000 & 1.000 & 100 \\
\hline
\end{tabular}

Table 2 lists the time consumed in the signal image coding and identification, in which image encoding and test is the time spent on one intrusion data, and training is the time consumed in 200 iterations.

Table 2. Time consumed.

\begin{tabular}{ccc}
\hline Image coding & Training & Test \\
\hline $0.76 \mathrm{~ms}$ & $272 \mathrm{~min}$ & $0.35 \mathrm{~s}$ \\
\hline
\end{tabular}

It can be seen from the results shown in Table 1 and Table 2, that the method proposed in this paper can achieve real-time and accurate intrusion events identification.

To further illustrate the performance of the method, the method proposed herein was compared with the method discussed above. It can be seen from Table 3 that our method can obtain the highest accuracy and identify relatively abundant intrusion event categories. This 
method does not need a rich understanding of signal knowledge. It can adaptively extract signal features, reduce human factors interference, and improve identified accuracy and efficiency.

Table 3. Comparison of different methods.

\begin{tabular}{|c|c|c|c|}
\hline Methods & $\begin{array}{l}\text { Accuracy } \\
(\%)\end{array}$ & $\begin{array}{l}\text { Category } \\
\text { numbers }\end{array}$ & Instruction category \\
\hline Wavelet decomposition and PNN [8] & 94 & 4 & $\begin{array}{l}\text { Footsteps of the human, Trench, } \\
\text { Climbing, Vehicle past }\end{array}$ \\
\hline EMD and BP neural network [9] & 95 & 3 & Little animal, Vehicle, Walker \\
\hline Frequency-time distribution [10] & 90 & 4 & Sloshing, Pulling, Knocking, Vibration \\
\hline AR and ZCR and SVM [11] & 87.14 & 6 & $\begin{array}{l}\text { Climbing, Knocking, Waggling, } \\
\text { Crashing, Kicking, Cutting }\end{array}$ \\
\hline $\begin{array}{l}\text { Spatial time-frequency spectrum and } \\
\text { DPN [13] }\end{array}$ & 97 & 7 & $\begin{array}{l}\text { Excavator operation, Concrete fence } \\
\text { breaking, Pedestrians, Tamping } \\
\text { operation, Ambient noise, Moving } \\
\text { train, Local wind }\end{array}$ \\
\hline RP and Inception-v3 (our method) & 99.7 & 6 & $\begin{array}{l}\text { Heavy rain, Light rain, Wind blowing, } \\
\text { Treading, Slapping, Impacting }\end{array}$ \\
\hline
\end{tabular}

\section{Conclusion}

In this paper, we built a distributed optical fiber dual Mach-Zehnder perimeter security system. For non-stationary nonlinear intrusion signals, we proposed an intrusion events identification method based on RP and Inception-v3. Identification accuracy of six representative intrusion events achieved $99.7 \%$, and in this application reached $0.35 \mathrm{~s}$ real-time detection. The results demonstrate the feasibility of the method.

The experimental results show that this method has a good effect on the identification of abnormal intrusion events. Nevertheless, in practical application, some intrusion events may be rare, and their datasets are relatively small. Thus, the current performance of the neural network model is affected. In the future we will study the identification scheme to deal with few-shot learning and also detect unknown events which are not in the training data.

\section{Acknowledgements}

This work was supported in part by National Natural Science Foundation of China under Grants 61205075.

\section{Declaration of Competing Interest}

The authors declare that there is no conflict of interest in this article.

\section{CRediT authorship contribution statement}

Chengang Lyu: Conceptualization, Project administration, Supervision, Resources, Writing - review \& editing. Jianying Jiang: Conceptualization, Investigation, Software, Data curation, Writing - original draft, Writing - review \& editing. Baihua Li: Conceptualization, Supervision, Writing - review \& editing. Ziqiang Huo: Conceptualization, Investigation, Data curation, Validation. Jiachen Yang: Project administration, Supervision, Resources.

\section{References}


[1] Y. Shi, H. Feng, and Z. Zeng, "Distributed fiber sensing system with wide frequency response and accurate location," Opt. Laser. Eng. 77, 219-224 (2016).

[2] I. A. Shcherbakov, Q. Sun, D. Liu, H. Liu, Y. He, J. Yuan, K. Xu, Q. Wang, A. V. Priezzhev, and V. I. Pustovoy, "Distributed disturbance sensor based on a novel Mach-Zehnder interferometer with a fiberloop," Proc. SPIE 6344, 1-7(2006).

[3] P. Ma, K. Liu, Z. Sun, J. Jiang, S. Wang, T. Xu, Z. Xu, and T. Liu, "Distributed single fiber optic vibration sensing with high frequency response and multi-points accurate location," Opt. Laser. Eng. 129(2020).

[4] J. C. Juarez, E. W. Maier, C. Kyoo Nam, and H. F. Taylor, "Distributed fiber-optic intrusion sensor system," J Lightwave Technol 23, 2081-2087 (2005).

[5] X. Bao and L. Chen, "Recent progress in distributed fiber optic sensors," Sensors. 12, 8601-8639 (2012).

[6] X. Liu, B. Jin, Q. Bai, Y. Wang, D. Wang, and Y. Wang, "Distributed Fiber-Optic Sensors for Vibration Detection," Sensors. 16, 1164- (2016).

[7] G. Allwood, G. Wild, and S. Hinckley, "Optical Fiber Sensors in Physical Intrusion Detection Systems: A Review," IEEE Sens. J. 16, 5497-5509 (2016).

[8] W. Liang, "Study on the fiber-optic perimeter sensor signal processor based on neural network classifier," in Proceedings of IEEE Conference on Electronic Measurement \& Instruments(IEEE, 2011), pp. 93-97.

[9] J. Li-hui, L. Xiang-ming, and Y. Ruo-yu, "Application of the HHT method to the airport fiber fence warning," in International Conference on Electronics, Communications and Control (IEEE, 2011), pp. 1337-1340.

[10] S. Liang, X. Sheng, S. Lou, Y. Feng, and K. Zhang, "Combination of Phase-Sensitive OTDR and Michelson Interferometer for Nuisance Alarm Rate Reducing and Event Identification," IEEE Photonics J. 8, 1-12 (2016).

[11] X.-D. Huang, H.-J. Zhang, K. Liu, and T.-G. Liu, "Fully modelling based intrusion discrimination in optical fiber perimeter security system," Opt Fiber Technol 45, 64-70 (2018).

[12] J. Wang, Y. Chen, S. Hao, X. Peng, and L. Hu, "Deep learning for sensor-based activity recognition: A survey," Pattern Recogn Lett 119, 3-11 (2019).

[13] Z. Wang, H. Zheng, L. Li, J. Liang, X. Wang, B. Lu, Q. Ye, R. Qu, and H. Cai, "Practical multi-class event classification approach for distributed vibration sensing using deep dual path network," Opt Express 27, 23682-23692 (2019).

[14] C. Lyu, Z. Huo, X. Cheng, J. Jiang, A. Alimasi, and H. Liu, "Distributed Optical Fiber Sensing Intrusion Pattern Recognition Based on GAF and CNN," J Lightwave Technol. (to be published).

[15] B. Kizlik, "Fibre optic distributed sensor in Mach-Zehnder interferometer configuration," in International Conference on Modern Problems of Radio Engineering, Telecommunications \& Computer Science (IEEE, 2002), pp. 128-130.

[16] M. S. Avila-Garcia, M. Bianchetti, R. Le Corre, A. Guevel, R. I. Mata-Chavez, J. M. Sierra-Hernandez, D. Jauregui-Vazquez, J. R. Reyes-Ayona, J. M. Estudillo-Ayala, and R. Rojas-Laguna, "High sensitivity strain sensors based on single-mode-fiber core-offset Mach-Zehnder interferometers," Opt. Laser. Eng. 107, 202206 (2018).

[17] Y. Zhao, H. Zhao, R.-q. Lv, and J. Zhao, "Review of optical fiber Mach-Zehnder interferometers with micro-cavity fabricated by femtosecond laser and sensing applications," Opt. Laser. Eng. 117, 7-20 (2019).

[18] J. P. Eckmann, S. O. Kamphorst, and D. Ruelle, "Recurrence Plots of Dynamical Systems," Europhys Lett 4, 973-977 (1987).

[19] M. Aboofazeli and Z. K. Moussavi, "Comparison of recurrence plot features of swallowing and breath sounds," Chaos, Solitons \& Fractals 37, 454-464 (2008).

[20] M. A. Alsaleh, "Detecting Chaos in Kuwait Exchange Rate Data," Journal of Mathematical Sciences 111, 3814-3819.

[21] L. Deng, X. Zhang, J. An, and Y. Cai, "Statistical properties of solar H $\alpha$ flare activity," Journal of Space Weather and Space Climate 7(2017).

[22] U. R. Acharya, O. Faust, N. Kannathal, T. Chua, and S. Laxminarayan, "Non-linear analysis of EEG signals at various sleep stages," Comput Methods Programs Biomed 80, 37-45 (2005).

[23] N. H. Packard, J. P. Crutchfield, J. D. Farmer, and R. S. Shaw, "Geometry from a Time Series," Phys Rev Lett 45, 712-716 (1980).

[24] T. Aparicio, E. F. Pozo, and D. Saura, "Detecting determinism using recurrence quantification analysis: Three test procedures," Journal of Economic Behavior \& Organization 65, 768-787 (2008).

[25] . N. Marwan, M. Carmenromano, M. Thiel, and J. Kurths, "Recurrence plots for the analysis of complex s ystems," Phys. Rep. 438, 237-329 (2007).

[26] Y. LeCun, Y. Bengio, and G. Hinton, "Deep learning," Nature 521, 436-444 (2015).

[27] P. Mamoshina, A. Vieira, E. Putin, and A. Zhavoronkov, "Applications of deep learning in biomedicine," Mol. Pharmaceutics 13, 1445-1454 (2016).

[28] D. Yu and L. Deng, "Deep learning and its applications to signal and information processing," IEEE Signal Proc Mag 28, 145-154 (2010).

[29] Y. Guo, Y. Liu, A. Oerlemans, S. Lao, S. Wu, and M. S. Lew, "Deep learning for visual understanding: A review," Neurocomputing 187, 27-48 (2016). 
[30] C. Szegedy, W. Liu, Y. Jia, P. Sermanet, S. Reed, D. Anguelov, D. Erhan, V. Vanhoucke, and A. Rabinovich, "Going deeper with convolutions," in IEEE Conference on Computer Vision and Pattern Recognition (CVPR) (IEEE, 2015), pp. 1-9.

[31] J. Yosinski, J. Clune, Y. Bengio, and H. Lipson, "How transferable are features in deep neural networks?," in Advances in Neural Information Process Systems (NIPS, 2014), pp. 3320-3328. 